\title{
Infusão contínua de propofol em gatos pré-medicados com cetamina-midazolam
}

[Continuous infusion of propofol after ketamine-midazolam premedication in cats]

\author{
A.L. Selmi ${ }^{1}$, J.P. Figueiredo ${ }^{2}$, G.M. Mendes $^{2}$, L.M.S. Lavor $^{2}$, P.M.L. Machado ${ }^{2}$ \\ ${ }^{1}$ Universidade Anhembi Morumbi \\ Rua Conselheiro Lafaiete, 64 - Brás \\ 03164-110 - São Paulo, SP \\ ${ }^{2}$ Faculdade de Agronomia e Medicina Veterinária - UnB - Brasília
}

\begin{abstract}
RESUMO
Estudaram-se os efeitos anestésicos e cardiorrespiratórios produzidos pela infusão contínua de propofol em gatos pré-medicados com a associação cetamina-midazolam. Catorze gatos adultos foram igualmente distribuídos em dois grupos (TX1 e TX3) aos quais administraram-se, pela via intramuscular, cetamina $(3,0 \mathrm{mg} / \mathrm{kg})$ e midazolam $(0,3 \mathrm{mg} / \mathrm{kg})$. Decorridos cinco minutos procedeu-se a indução anestésica pela administração intravenosa de propofol $(5,0 \mathrm{mg} / \mathrm{kg})$, imediatamente seguida pela infusão contínua do agente hipnótico nas doses de 0,1 ou $0,3 \mathrm{mg} / \mathrm{kg} / \mathrm{min}$, aos animais de TX1 e TX3, respectivamente. Foram mensuradas as freqüências cardíaca e respiratória, temperatura retal, saturação de oxihemoglobina, concentração exalada de dióxido de carbono e pressão arterial. Em TX3 observou-se manutenção de adequado plano anestésico, enquanto que os animais do TX1 apresentaram-se sedados. Houve decréscimo acentuado da freqüência cardíaca, pressão arterial e elevação da concentração de dióxido de carbono exalado no TX3. Conclui-se que o emprego de propofol na dose de infusão de $0,3 \mathrm{mg} / \mathrm{kg} / \mathrm{min}$ em gatos pré-medicados com cetamina-midazolam produz anestesia satisfatória, bradicardia, depressão da função respiratória e pressão arterial.
\end{abstract}

Palavras-chave: gato, propofol, infusão contínua, cetamina, midazolam

\begin{abstract}
Anesthetic and cardiorespiratory effects of two different infusion rates of propofol were studied in cats premedicated with ketamine-midazolam. Fourteen cats were assigned to one of the two groups (TX1 or TX3). Ketamine $(3.0 \mathrm{mg} / \mathrm{kg})$ and midazolam $(0.3 \mathrm{mg} / \mathrm{kg})$ were administered intramuscularly. After 5 minutes, anesthesia was induced by propofol $(5.0 \mathrm{mg} / \mathrm{kg})$ and maintained by a continuous infusion of propofol $(0.1$ and $0.3 \mathrm{mg} / \mathrm{kg} / \mathrm{min}, T X 1$ and $T X 3$, respectively). Heart and respiratory rate, rectal temperature, oxygen hemoglobin saturation, end-tidal carbon dioxide and arterial pressure were recorded. Adequate anesthesia was observed in TX3, while animals in TX1 were only lightly sedated. A greater decrease in heart rate, arterial pressure and elevation of end-tidal dioxide carbon was observed in TX3 compared to TX1. It was concluded that administration of propofol at infusion rate of $0.3 \mathrm{mg} / \mathrm{kg} / \mathrm{min}$ provides satisfatory anesthesia, but it results in bradycardia, depression of respiratory function and arterial pressure.
\end{abstract}

Keywords: cat, propofol, continuous infusion, ketamine, midazolam

Recebido para publicação em 16 de maio de 2003

Recebido para publicação, após modificações, em 9 de março de 2004

E-mail: andre_selmi@yahoo.com.br 


\section{INTRODUÇÃO}

A utilização de protocolos intravenosos injetáveis tem sido preconizada na recente prática anestésica (Beths et al., 2001; Muir e Gadawski, 2002). Acredita-se que a anestesia intravenosa produza efeitos cardiovasculares menos pronunciados que o emprego de anestesia inalatória (Keegan e Greene, 1993), e ainda evita a contaminação ambiente por gases anestésicos (Beths et al., 2001). As características farmacocinéticas do propofol, como rápida eliminação e grande volume de distribuição, fazem com que esse agente anestésico seja considerado uma importante alternativa nos diversos protocolos anestésicos intravenosos (Glen, 1980; Muir e Gadawski, 2002). Em gatos, o propofol promove hipotensão arterial, podendo ou não alterar a freqüência cardíaca, além de causar depressão respiratória (Muir e Gadawski, 2002).

Diferentemente do cão, ainda são escassas as informações relativas à farmacocinética do propofol em felinos (Glen, 1980; Matot et al., 1993; Beths et al., 2001). Isso inviabiliza o emprego de protocolos de anestesia intravenosa com base na concentração plasmática de propofol em felinos e torna essencial o estudo de protocolos de infusão baseados na dose de infusão do anestésico intravenoso (Ilkiw e Pascoe, 2003).

A administração conjunta de cetamina e benzodiazepínicos representa uma das associações mais amplamente empregadas para anestesia e pré-medicação em pequenos animais (Hellyer et al., 1991; Ilkiw et al., 1998), proporcionando segurança, mínimos efeitos depressores, além de rápida recuperação anestésica (Hellyer et al., 1991). Apesar de a administração do midazolam, benzodiazepínico hidrossolúvel, freqüentemente produzir alterações de comportamento tais como excitação, vocalização e agressividade à contenção em gatos (Ilkiw et al., 1996), esse fármaco demonstra maior potência do que o diazepam, o agente desse grupo mais freqüentemente empregado na anestesia veterinária (Ilkiw et al., 1998).

Este trabalho objetivou caracterizar os efeitos anestésicos e cardiorrespiratórios produzidos pela administração de duas doses de infusão de propofol em gatos pré-medicados com cetaminamidazolam.

\section{MATERIAL E MÉTODOS}

Foram utilizados 14 gatos, machos e fêmeas sem raça definida, adultos, idade entre 2,0 e 3,7 anos e média de peso de $2,8 \pm 0,9 \mathrm{~kg}$. Todos os animais foram considerados clinicamente sadios após realização de exames clínico-laboratoriais. Foram mantidos em jejum alimentar e hídrico por 12 e 6 horas, respectivamente, antes da realização dos procedimentos anestésicos.

Os valores referentes à pressão arterial sistólica (PAS), diastólica (PAD) e média (PAM), saturação parcial de oxigênio $\left(\mathrm{SpO}_{2}\right)$ e a concentração de dióxido de carbono ao final da expiração $\left(\mathrm{EtCO}_{2}\right)$ foram obtidos por meio de monitor multiparamétrico. A freqüência cardíaca (FC) foi mensurada por meio de estetoscópio; a freqüência respiratória (FR) foi determinada pelo número de excursões torácicas durante um minuto e a temperatura retal foi registrada por meio de termômetro digital. Todas as variáveis foram avaliadas no momento anterior à aplicação da associação cetamina-midazolam (M0), cinco minutos após a pré-medicação e imediatamente antes da indução anestésica por propofol (M1), 10 minutos após o início da infusão de propofol (M2) e consecutivamente, em intervalos de 15 minutos, até 90 minutos após a pré-medicação (M3 a M7).

Após determinação dos valores basais para cada variável cardiorrespiratória de interesse, realizou-se a administração conjunta de cetamina, na dose de $3,0 \mathrm{mg} / \mathrm{kg}$, e midazolam, na dose de $0,3 \mathrm{mg} / \mathrm{kg}$, pela via intramuscular. Após cinco minutos, fez-se a indução anestésica com propofol na dose de $5,0 \mathrm{mg} / \mathrm{kg}$, pela via intravenosa. Imediatamente após a indução anestésica, os animais foram igualmente divididos em dois grupos de forma aleatória; no primeiro grupo (TX1), administrou-se $0,1 \mathrm{mg} / \mathrm{kg} / \mathrm{min}$ de propofol e no segundo (TX3), propofol na dose de infusão de $0,3 \mathrm{mg} / \mathrm{kg} / \mathrm{min}$, pela via intravenosa. Durante todos os procedimentos anestésicos, foi fornecido oxigênio a $100 \%(300 \mathrm{ml} / \mathrm{kg} / \mathrm{min})$ por meio de máscara facial vedada, acoplada em circuito anestésico Baraka. 
A qualidade e profundidade anestésica foram avaliadas periodicamente durante a manutenção anestésica dos animais (rotação do globo ocular, movimentação voluntária dos animais, reflexo palpebral, reflexo corneal, reflexo pedal, reflexo auricular), conforme relatado por Trim (1991) para a espécie.

Usou-se a análise de variância para medidas repetidas, considerando os momentos de avaliação para cada grupo, seguida pelo teste de Tukey. O grau de significância estabelecido foi de $5 \%$.

\section{RESULTADOS}

Observaram-se sedação e decúbito após a administração de cetamina-midazolam em quatro animais (4/7) do TX1 e em três animais (3/7) do TX3. Um animal do TX1 e dois de TX3 apresentaram leve excitação após a prémedicação. A indução anestésica foi desprovida de incidentes adversos (apnéia, excitação, êmese) em todos os animais.

De modo geral, os animais do TX1 apresentaram leve sedação durante o período de infusão contínua de propofol, sendo observados movimentos suaves de membros, língua $\mathrm{e}$ orelhas, além de presença de nistagmo e manutenção dos reflexos palpebral, pedal, auricular e corneal. No TX3, exceto pelos 20 minutos iniciais, quando houve a manutenção do reflexo corneal, todas as outras variáveis clínicas avaliadas foram abolidas após a indução anestésica e infusão de propofol.

Com relação às variáveis cardiovasculares (Tab. 1), ocorreu acréscimo significativo da freqüência cardíaca após a pré-medicação em M1 e M3 em TX3 e TX1, respectivamente, e decréscimo após M4 e M5 em TX1 e TX3, respectivamente. Em ambos os grupos houve diminuição dos valores médios de pressão arterial sistólica, diastólica e média, entretanto o efeito foi mais intenso e prolongado no TX3.

Tabela 1. Variáveis cardiovasculares em gatos submetidos à pré-medicação com cetamina-midazolam, indução por propofol e manutenção anestésica com duas doses de infusão contínua de propofol

\begin{tabular}{|c|c|c|c|c|c|c|c|c|c|}
\hline \multirow{2}{*}{ Variável } & \multirow{2}{*}{ Grupo } & \multirow{2}{*}{ M0 } & \multicolumn{7}{|c|}{ Momento após a pré-medicaAção } \\
\hline & & & M1 & M2 & M3 & M4 & M5 & M6 & M7 \\
\hline $\mathrm{FC}$ & TX1 & $165,2 \pm 12,4$ & $184,4 \pm 11,7 \mathrm{~b}$ & $202,2 \pm 22,7 \mathrm{Aa}$ & $160,1 \pm 15,3 a$ & $146,8 \pm 24,2 \mathrm{a}$ & $132,2 \pm 13,7 \mathrm{~A}$ & $127,6 \pm 11,6 \mathrm{~A}$ & $127,6 \pm 14,4 \mathrm{~A}$ \\
\hline (bpm) & TX3 & $160,5 \pm 9,5$ & $204,7 \pm 20,6 \mathrm{Aa}$ & $168,4 \pm 40,7 b$ & $139,2 \pm 30,0 b$ & $127,0 \pm 25,3 \mathrm{Ab}$ & $123,8 \pm 23,2 \mathrm{~A}$ & $119,2 \pm 21,8 \mathrm{~A}$ & $119,2 \pm 21,2 \mathrm{~A}$ \\
\hline PAS & TX1 & $111,0 \pm 4,7$ & $110,6 \pm 5,6$ & $112,8 \pm 9,6 a$ & $109,2 \pm 13,4 \mathrm{a}$ & $100,6 \pm 5,8 \mathrm{a}$ & $98,2 \pm 6,1 \mathrm{Aa}$ & $98,0 \pm 6,1 \mathrm{Aa}$ & $94,2 \pm 6,1 \mathrm{Aa}$ \\
\hline$(\mathrm{mmHg})$ & TX3 & $113,5 \pm 5,2$ & $109,0 \pm 9,0$ & $103,8 \pm 25,5 \mathrm{Ab}$ & $90,2 \pm 8,5 \mathrm{Ab}$ & $89,0 \pm 11,8 \mathrm{Ab}$ & $85,4 \pm 14,5 \mathrm{Ab}$ & $88,6 \pm 12,6 \mathrm{Ab}$ & $88,6 \pm 16,8 \mathrm{Ab}$ \\
\hline PAD & TX1 & $83,7 \pm 0,9$ & $88,2 \pm 11,8$ & $84,2 \pm 19,2 \mathrm{a}$ & $80,8 \pm 19,6 \mathrm{a}$ & $71,2 \pm 22,3 \mathrm{a}$ & $64,8 \pm 17,8 \mathrm{~A}$ & $63,4 \pm 18,8 \mathrm{~A}$ & $64,0 \pm 11,5 \mathrm{~A}$ \\
\hline$(\mathrm{mmHg})$ & TX3 & $84,0 \pm 4,3$ & $82,2 \pm 11,7$ & $75,0 \pm 22,0 \mathrm{Ab}$ & $69,8 \pm 9,7 \mathrm{Ab}$ & $65,2 \pm 14,7 \mathrm{Ab}$ & $62,2 \pm 18,6 \mathrm{~A}$ & $63,4 \pm 18,3 \mathrm{~A}$ & $63,2 \pm 17,6 \mathrm{~A}$ \\
\hline PAM & TX1 & $93,7 \pm 2,8$ & $94,6 \pm 7,3$ & $90,0 \pm 16,3 \mathrm{a}$ & $95,4 \pm 13,7 \mathrm{a}$ & $85,2 \pm 8,3 \mathrm{a}$ & $82,1 \pm 15,4 \mathrm{Aa}$ & $80,7 \pm 12,6 \mathrm{~A}$ & $81,5 \pm 11,4 \mathrm{Aa}$ \\
\hline$(\mathrm{mmHg})$ & TX3 & $94,5 \pm 3,3$ & $96,2 \pm 17,7$ & $80,0 \pm 22,9 \mathrm{Ab}$ & $71,0 \pm 10,8 \mathrm{Ab}$ & $70,2 \pm 18,4 \mathrm{Ab}$ & $71,7 \pm 15,8 \mathrm{Ab}$ & $72,4 \pm 15,3 \mathrm{~A}$ & $71,8 \pm 12,4 \mathrm{Ab}$ \\
\hline
\end{tabular}

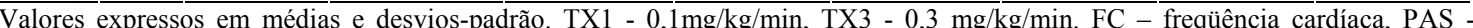
pressão arterial sistólica, PAD - pressão arterial diastólica, PAM - pressão arterial média. M0 - momento pré-anestésico; M1- 5 minutos após pré-medicação e previamente à indução anestésica por propofol; M2 - 10 minutos após início da infusão de propofol; M3 a M7 - intervalos regulares subseqüentes de 15 minutos.

Letra A na linha indica valor diferente de M0 (teste Tukey; $\mathrm{P}<0,05$ ).

Letras minúsculas distintas na coluna, dentro de cada variável, indicam diferenças entre grupos (teste Tukey; $\mathrm{P}<0,05$ ).

A freqüência respiratória diminuiu de modo similar nos dois grupos, entretanto o aumento nos níveis médios de dióxido de carbono expirado foi maior em TX3 após a indução anestésica e a subseqüente manutenção por propofol (Tab. 2). Não houve alterações nos valores de saturação de oxihemoglobina dentre e entre os grupos. A temperatura retal decresceu em M6 em ambos os grupos. 
Tabela 2. Variáveis respiratórias e temperatura retal em gatos submetidos à pré-medicação com cetaminamidazolam, indução por propofol e manutenção anestésica com duas doses de infusão contínua de propofol

\begin{tabular}{|c|c|c|c|c|c|c|c|c|c|}
\hline \multirow{2}{*}{ Variável } & \multirow{2}{*}{ Grupo } & \multirow{2}{*}{10} & \multicolumn{7}{|c|}{ Momento após a pré-medicação } \\
\hline & & & M1 & M2 & M3 & M4 & M5 & M6 & M7 \\
\hline \multirow{2}{*}{$\begin{array}{l}\text { FR } \\
(\mathrm{mov} / \mathrm{min})\end{array}$} & & $32+33$ & 0,0 & $2+3$ & & & 28 & & \\
\hline & & & & 22 & & & & & \\
\hline \multirow{2}{*}{$\mathrm{SpO}_{2}(\%)$} & T) & & & & & & & & \\
\hline & TX3 & & & & & & & 98,0 & $97,3 \pm 1,8$ \\
\hline \multirow{2}{*}{$\begin{array}{l}\mathrm{EtCO}_{2} \\
(\mathrm{mmHg})\end{array}$} & & o & $6 \mathrm{~A}$ & 54 & 56,2 &, $0 \mathrm{Ab}$ & $59,3 \pm 7,1 \mathrm{~A}$ & $57,4 \pm 9,8 \mathrm{Ab}$ & $57,5 \pm 6,5 \mathrm{Ab}$ \\
\hline & TX3 & & 56,2 & $67,5 \pm \varepsilon$ & 70,3 & $4 \mathrm{Aa}$ & $67,5 \pm 9,9 \mathrm{~A}$ & $69,8 \pm 12,5 \mathrm{Aa}$ & $71,2 \pm 11,7 \mathrm{Aa}$ \\
\hline \multirow{2}{*}{$\operatorname{TR}\left({ }^{\circ} \mathrm{C}\right)$} & TX1 & & & $37,8 \pm 0,8$ & & & & $37,2 \pm 0,6 \mathrm{~A}$ & $37,0 \pm 1,3 \mathrm{~A}$ \\
\hline & TX3 & $38,6 \pm 0,8$ & $38,4 \pm 0,5$ & $38,3 \pm 0,5$ & $37,7 \pm 0,4$ & $37,6 \pm 0,3$ & $37,3 \pm 1,3$ & $37,1 \pm 1,4 \mathrm{~A}$ & $37,0 \pm 1,2 \mathrm{~A}$ \\
\hline
\end{tabular}

Valores expressos em médias e desvios-padrão. TX1 - 0,1 $\frac{\mathrm{mg} / \mathrm{kg} / \mathrm{min}, \mathrm{TX} 3-0,3 \mathrm{mg} / \mathrm{kg} / \mathrm{min}, \mathrm{FR} \text { - freqüência respiratória, SpO }}{2}$ saturação de oxihemoglobina, $\mathrm{EtCO}_{2}$ - concentração de dióxido de carbono exalado, TR - temperatura retal. M0 - momento préanestésico; M1- 5 minutos após pré-medicação e previamente à indução anestésica por propofol; M2 - 10 minutos após início da infusão de propofol; M3 a M7 - intervalos regulares subseqüentes de 15 minutos.

Letra A na linha indica valor diferente de M0 (teste Tukey; $\mathrm{P}<0,05$ ).

Letras minúsculas distintas na coluna, dentro de cada variável, indicam diferenças entre grupos (teste Tukey; $\mathrm{P}<0,05$ ).

\section{DISCUSSÃO}

A ocorrência de excitação após a administração de midazolam-cetamina tem sido relatada em gatos (Ilkiw et al., 1998), sendo considerada efeito característico do midazolam na espécie (Ilkiw et al., 1996). A prevalência desse fenômeno observada por Ilkiw et al. (1998), após a administração intravenosa de midazolamcetamina, foi mais elevada $(66 \%)$ do que a observada neste estudo, fato possivelmente relacionado às diferentes vias de administração empregadas.

O aumento transitório da freqüência cardíaca, logo após a administração de cetaminamidazolam, decorreu da ação simpatomimética dos agentes dissociativos, como foi observado por Cullen e Reynoldson (1997) em cães prémedicados com tiletamina-zolazepam e anestesiados por propofol.

Ilkiw e Pascoe (2003) relataram que o emprego de cetamina em infusão contínua produz significativa redução na dose de infusão mínima de propofol em gatos. Acredita-se que efeito semelhante tenha sido observado neste estudo, entretanto a magnitude da potencialização do propofol pela pré-medicação por midazolamcetamina não foi suficiente para produzir anestesia satisfatória no TX1.

Segundo Glen (1980), o maior volume de distribuição e a menor taxa de eliminação do propofol são algumas diferenças farmacocinéticas observadas entre a espécie felina e os demais animais. Interessante notar que essa diferença demonstra a necessidade de estudos clínicos e farmacocinéticos envolvendo o emprego do propofol em felinos, pois a dose de infusão de propofol necessária para a produção de anestesia em gatos (Ilkiw e Pascoe, 2003) é consideravelmente menor $(0,15 \mathrm{mg} / \mathrm{kg} / \mathrm{min})$ quando comparada à dose de infusão mínima deste agente $(0,44 \mathrm{mg} / \mathrm{kg} / \mathrm{min})$ em cães (Keegan et al., 1993).

As alterações respiratórias observadas nos gatos deste estudo corroboram com os resultados obtidos em cães (Cullen e Reynoldson, 1997), nos quais a pré-medicação com a associação tiletamina-zolazepam e anestesia com propofol produziu redução da freqüência respiratória, hipoxemia e hipercapnia.

A necessidade da instituição de suporte ventilatório tem sido enfatizada durante a anestesia intravenosa pelo propofol (Keegan et al., 1993), entretanto a ventilação espontânea foi mantida nos gatos deste estudo pela impossibilidade de intubação dos animais no TX1. Além disso, a adoção desse procedimento poderia modificar os efeitos cardiovasculares observados em ambos os grupos, segundo Muir e Gadawski (2002).

A administração de oxigênio por meio de máscara tem sido recomendada em felinos (Taylor, 1991). Neste estudo, esse procedimento foi eficaz na manutenção dos valores de 
saturação de oxihemoglobina e eliminação de hipoxemia. Entretanto, os valores expirados de dióxido de carbono obtidos foram provavelmente subestimados, pois pode ter ocorrido diluição do dióxido de carbono expirado pelo fluxo de oxigênio empregado (Trim, 1991).

Segundo Muir e Gadawski (2002), em gatos prémedicados com romifidina e mantidos em ventilação mecânica, o propofol, administrado na dose de infusão de $0,16 \mathrm{mg} / \mathrm{kg} / \mathrm{min}$, não produz alterações hemodinâmicas significativas. Não obstante, o emprego desse fármaco em infusão contínua resulta no decréscimo significativo da freqüência cardíaca e da pressão arterial, possivelmente pela inibição dose-dependente de mecanismos vasopressores medulares com conseqüente ação vasodilatadora, e, segundo Cullen e Reynoldson (1997), pode ainda estar relacionado à elevação dos níveis de dióxido de carbono, como observado neste estudo.

A ocorrência de hipotermia durante a realização de procedimentos anestésicos em felinos, como observada neste estudo, tem sido freqüentemente relatada (Taylor, 1991) e reflete a alta relação entre a superfície corpórea e o volume, nessa espécie (Trim, 1991).

\section{CONCLUSÕES}

A pré-medicação com midazolam e cetamina pela via intramuscular seguida pela infusão contínua de propofol na dose de $0,3 \mathrm{mg} / \mathrm{kg} / \mathrm{min}$ promove plano anestésico adequado em gatos, entretanto acompanhado de depressão cardiorrespiratória.

\section{REFERÊNCIAS BIBLIOGRÁFICAS}

BETHS, T.; GLEN, J.B.; REID, J. et al. Evaluation and optimization of a targetcontrolled infusion system for administering propofol to dogs as part of a total intravenous anaesthetic technique during dental surgery. Vet. Rec., v.148, p.198-203, 2001.
CULLEN, L.K.; REYNOLDSON, J.A. Effects of tiletamine-zolazepam premedication on propofol anaesthesia in dogs. Vet. Rec., v.140, p.363-366, 1997.

GLEN, J.G. Animal studies of the anaesthetic activity of ICI 35 868. Brit. J. Anaesth., v.52, p.731-742, 1980.

HELLYER, P.W.; FREEMAN, L.C.; HUBBELL, J.A.E. Induction of anesthesia with diazepam-ketamine and midazolam-ketamine in greyhounds. Vet. Surg., v.20, p.143-147, 1991.

ILKIW, J.E.; PASCOE, P.J. Cardiovascular effects of propofol alone or in conbination with ketamine for total intravenous anesthesia in cats. Am. J. Vet. Res., v.64, p.913-917, 2003.

ILKIW, J.E.; SUTER, C.; MCNEAL, D. et al. The optimal intravenous dose of midazolam after intravenous ketamine in healthy cats. J. Vet. Pharmacol. Therap., v.21, p.54-61, 1998.

ILKIW, J.E.; SUTER, C.M.; FARVER, T.B. et al. The behaviour of awake cats following intravenous and intramuscular administration of midazolam. J. Vet. Pharmacol. Therap., v.19, p.205-216, 1996.

KEEGAN, R.D; $\quad$ GREENE, S.A. Cardiopulmonary effects of a continuous twohours propofol infusion in dogs: Comparison with isoflurane anesthesia. Vet. Surg., v.22, p.537-543, 1993.

MATOT, I.; NEELY, C.F.; KATZ, R.Y. et al. Pulmonary uptake of propofol in cats. Anesthesiology, v.78, p.1157-1165, 1993.

MUIR III, W.W.; GADAWSKI, J.E. Cardiovascular effects of a higher dose of romifidine in propofol-anesthetized cats. Am. J. Vet. Res., v.63, p.1241-1246, 2002.

TAYLOR, P.M. Accidents and emergencies. In: HALL, L.W.; TAYLOR, P.M. Anaesthesia of the cat. London: Bailliere Tindall, 1994. p.249-273.

TRIM, J.E. Monitoring the anaesthetized cat. In: HALL, L.W.; TAYLOR, P.M. Anaesthesia of the cat. London: Bailliere Tindall, 1994. p.194-223. 